\title{
Some Problems on Best Approximation in Orlicz Spaces*
}

\author{
Garidi Wu ${ }^{1}$, Dongyue Guan ${ }^{2}$ \\ ${ }^{1}$ College of Mathematics Science, Inner Mongolia Normal University, Huhhot, China \\ ${ }^{2}$ College of Natural Science, Inner Mongolia Agricultural University, Huhhot, China \\ Email: wgrd@imnu.edu.cn, 494777843@qq.com
}

Received January 17, 2012; revised February 19, 2012; accepted February 26, 2012

\begin{abstract}
In this paper we studied some problems on best approximation in Orlicz spaces, for which the approximating sets are Haar subspaces, the result of this paper can be considered as the extension of the classical corresponding result.
\end{abstract}

Keywords: Chebyshev System; Haar Subspace; Orlicz Space; Best Approximation

\section{Introduction}

Let $Q$ be a compact Hausdorff space, $C(Q)$ be all the continuous functions on $Q$. There are at least $n$ points on $Q,\left\{\varphi_{1}(t), \cdots, \varphi_{n}(t)\right\} \subset C(Q)$. Define $\left\{\varphi_{1}(t), \cdots, \varphi_{n}(t)\right\}$ as $n$ order Chebyshev system if for arbitrary vector $\left(c_{1}, \cdots, c_{n}\right) \neq(0, \cdots, 0)$,

$$
p(t)=\sum_{i=1}^{n} c_{i} \varphi_{i}(t)
$$

has at most $n-1$ zero points on $Q$ [1].

Define the linear subspace

$$
H=\operatorname{span}\left\{\varphi_{1}(t), \cdots, \varphi_{n}(t)\right\}
$$

which is spanned by $n$ order Chebyshev system as a Haar subspace of $C(Q)$ [1].

In this paper, let $M(u)$ and $N(v)$ be mutually complementary $N$ function. The definition and properties of $N$ function can be seen in [2]. The Orlicz space $L_{M}^{*}(Q)$ corresponding to the $N$ function $M(u)$ consists of all Lebesgue measurable functions $\{u(x)\}$ on $Q$, of which the Orlicz norm

$$
\|u\|_{M}=\sup _{\rho(v, N) \leq 1}\left|\int_{Q} u(x) v(x) \mathrm{d} x\right|
$$

is finite, here

$$
\rho(v, N)=\int_{Q} N(v(x)) \mathrm{d} x
$$

is the modulus of $v(x)$ corresponding to $N(v)$. According to [2], the Orlicz norm (1.1) can also be calculated by

$$
\|u\|_{M}=\inf _{\alpha>0} \frac{1}{\alpha}\left(1+\int_{Q} M(\alpha u(x)) \mathrm{d} x\right),
$$

and there exists an $\alpha>0$, satisfying

$$
\int_{Q} N(p(\alpha|u(x)|)) \mathrm{d} x=1,
$$

shch that

$$
\|u\|_{M}=\frac{1}{\alpha}\left(1+\int_{Q} M(\alpha u(x)) \mathrm{d} x\right),
$$

here $p(u)$ is the derivative of $M(u)$ on the right. Equivalent to the Orlicz norm (1.1), in Orlicz space $L_{M}^{*}(Q)$, the Luxemburg norm is defined by

$$
\|u\|_{(M)}=\inf \left\{\alpha>0: \int_{Q} M\left(\frac{u(x)}{\alpha}\right) \mathrm{d} x \leq 1\right\} .
$$

In the sequel $L_{M}^{*}$ and $L_{(M)}^{*}$ will denote the Orlicz space with Orlicz norm (1.1) and the Luxemburg norm (1.3) respectively.

It is well known that

$$
C(Q) \subset L_{p}(Q) \subset L_{M}^{*}(Q)\left(L_{(M)}^{*}(Q)\right)(p>1) .
$$

\section{Main Results}

Now we choose $Q=[a, b]$ and

$$
H=\operatorname{span}\left\{\varphi_{1}(t), \cdots, \varphi_{n}(t)\right\}
$$

is a Haar subspace of $C[a, b]$, then we obtain

Theorem 1. Let $M(u)$ be $N$ function satisfying $\Delta_{2}$ condition, of which the derivative on the right $p(u)$ is continuous and strictly monotone increasing, $f(t) \in C[a, b], \quad f(t) \notin \bar{H}$, if $p_{n}^{*}(t) \in H$ is the best approximator in the mean of $f(t)$ in $H$ for the Orlicz norm \|\|$_{M}$ or the Luxemburg norm \|\|$_{(M)}$, then there exist at least $n$ different zero points of $f(t)-p_{n}^{*}(t)$ in $(a, b)$.

In order to prove this theorem, first we state the fol- 
lowing two lemmas.

Lemma 1. [3-5]. Let $M(u)$ be $N$ function satisfying $\Delta_{2}$ condition, of which the derivative on the right $p(u)$ is continuous and strictly monotone increasing, $F$ is a linear subspace of $L_{(M)}^{*}[a, b], f(t) \in L_{(M)}^{*}[a, b] \backslash \bar{F}$, then $g_{0}(t) \in F$ is the best approximator in the mean of $f(t)$ in $F$ for the Luxemburg norm $\|\cdot\|_{(M)}$, if and only if for arbitrary function $g(t) \in F$,

$\int_{a}^{b} g(t) p\left(\frac{\left|f(t)-g_{0}(t)\right|}{\left\|f-g_{0}\right\|_{(M)}}\right) \operatorname{sgn}\left(f(t)-g_{0}(t)\right) \mathrm{d} t=0$ holds true.

Lemma 2. [4,5]. Under the conditions of lemma 1 , $g_{0}(t) \in F$ is the best approximator in the mean of $f(t)$ in $F$ for the Orlicz norm $\|\cdot\|_{M}$, if and only if for arbitrary function $g(t) \in F$,

$\int_{a}^{b} g(t) p\left(k\left|f(t)-g_{0}(t)\right|\right) \operatorname{sgn}\left(f(t)-g_{0}(t)\right) \mathrm{d} t=0$ holds true, here $k$ satisfies

$$
\int_{a}^{b} N\left(p\left(k\left|f(t)-g_{0}(t)\right|\right)\right) \mathrm{d} t=1 .
$$

Proof of Theorem 1. We prove first the case of the Luxemburg norm. Here we take reduction to absurdity. Assume there exist at most $n-1$ different zero points $t_{1}, \cdots, t_{r}(r \leq n-1)$ of $f(t)-p_{n}^{*}(t)$ in $(a, b)$. Based on $t_{1}, \cdots, t_{r}$, we choose $n-1$ points in $(a, b)$, such that $a=\xi_{0}<\xi_{1}<\cdots<\xi_{r}<\cdots<\xi_{n-1}<\xi_{n}=b$, here $\xi_{j}=t_{j}$, $j=1, \cdots, r$. From lemma 1 we get

$$
\begin{gathered}
\int_{a}^{b} \varphi_{j}(t) p\left(\frac{\left|f(t)-p_{n}^{*}(t)\right|}{\left\|f-p_{n}^{*}\right\|_{(M)}}\right) . \\
\operatorname{sgn}\left(f(t)-p_{n}^{*}(t)\right) \mathrm{d} t=0, j=1, \cdots, n .
\end{gathered}
$$

For $f(t)-p_{n}^{*}(t) \in C[a, b]$, the above can be deduced as following

$$
\begin{aligned}
& \sum_{i=1}^{n} \sigma_{i} \int_{\xi_{i-1}}^{\xi_{i}} \varphi_{j}(t) p\left(\frac{\left|f(t)-p_{n}^{*}(t)\right|}{\left\|f-p_{n}^{*}\right\|_{(M)}}\right) d t \\
& =\sum_{i=1}^{n} \sigma_{i} \psi_{i}\left(\varphi_{j}\right)=0, j=1, \cdots, n,
\end{aligned}
$$

here every $\sigma_{i}=1$ or -1 ,

$$
\begin{aligned}
& \psi_{i}\left(\varphi_{j}\right)=\int_{\xi_{i-1}}^{\xi_{i}} \varphi_{j}(t) p\left(\frac{\left|f(t)-p_{n}^{*}(t)\right|}{\left\|f-p_{n}^{*}\right\|_{(M)}}\right) \mathrm{d} t, \\
& i=1, \cdots, n, j=1, \cdots, n .
\end{aligned}
$$

According to the theory of system of linear equations, we have that $\operatorname{det}\left(\psi_{i}\left(\varphi_{j}\right)\right)_{i, j=1}^{n}=0$, hence the transposed system of equations $\sum_{j=1}^{n} c_{j} \psi_{i}\left(\varphi_{j}\right)=0, i=1, \cdots, n$ also has a nonzero solution $\left(c_{1}, \cdots, c_{n}\right)$. Set $p_{n}(t)=\sum_{j=1}^{n} c_{j} \varphi_{j}(t)$, then $p_{n}(t) \neq 0$ for some $t$. On the other hand,

$$
\begin{aligned}
\psi_{i}\left(p_{n}\right) & =\int_{\xi_{i-1}}^{\xi_{i}} p_{n}(t) p\left(\frac{\left|f(t)-p_{n}^{*}(t)\right|}{\left\|f-p_{n}^{*}\right\|_{(M)}}\right) \mathrm{d} t \\
& =\sum_{j=1}^{n} c_{j} \int_{\xi_{i-1}}^{\xi_{i}} \varphi_{j}(t) p\left(\frac{\left|f(t)-p_{n}^{*}(t)\right|}{\left\|f-p_{n}^{*}\right\|_{(M)}}\right) \mathrm{d} t \\
& =\sum_{j=1}^{n} c_{j} \psi_{i}\left(\varphi_{j}\right)=0, i=1, \cdots, n .
\end{aligned}
$$

Since $p(u)$ is the derivative of $N$ function $M(u)$ on the right, according to the properties of $N$ function (see [2]) and the hypothesis of $p(u)$, we obtain

$$
p\left(\frac{\left|f(t)-p_{n}^{*}(t)\right|}{\left\|f-p_{n}^{*}\right\|_{(M)}}\right)>0, t \in\left(\xi_{i-1}, \xi_{i}\right), i=1, \cdots, n .
$$

The above shows that there exist zero points of the continuous function $p_{n}(t)$ in every interval $\left(\xi_{i-1}, \xi_{i}\right)$ $(i=1, \cdots, n)$, that is to say, $p_{n}(t)$ has at least $n$ different zero points in interval $(a, b)$. Since $\left\{\varphi_{1}(t), \cdots, \varphi_{n}(t)\right\}$ is $n$ order Chebyshev system, we get $p_{n}(t) \equiv 0$, Together with the previous result, we get a contradiction.

In an analogous way, following lemma 2 we can also prove the case of the Orlicz norm.

In the sequel we choose $Q=[-1,1], \varphi_{j}(t)=t^{j-1}$, $j=1, \cdots, n$, then the Haar subspace of $C[-1,1]$ is $H=\operatorname{span}\left\{1, t, \cdots, t^{n-1}\right\}$, consists of all algebraic polynomials of order not larger than $n-1$. For $f(t)=t^{n}$, in order to solve the problem of best approximation of $f(t)$ with $H$ in Orlicz space, actually we just need to consider the problem of the minimal norm of monic polynomials of order $n$ in Orlicz space, that is, to consider the extreme value problems as following

$$
\begin{aligned}
& \min _{a_{j}}\left\|t^{n}+\sum_{j=1}^{n} a_{j} t^{n-j}\right\|_{M} ; \\
& \min _{a_{j}}\left\|t^{n}+\sum_{j=1}^{n} a_{j} t^{n-j}\right\|_{(M)} .
\end{aligned}
$$

The similar problems in $L_{p}$ space has not been completely solved except $p=1,2,+\infty$ (see [6]). In Orlicz spaces the problems have not been studied yet. Here we obtain

Theorem 2. Let $M(u)$ be $N$ function satisfying $\Delta_{2}$ condition, its graph do not contain any straight line segment, its derivative on the right $p(u)$ be continuous and strictly monotone increasing, then 
1) The extreme value problems (2.1) and (2.2) have unique solution respectively, that is, there exist unique group $a_{1, M}, \cdots, a_{n, M}$ and $a_{1, M}^{\prime}, \cdots, a_{n, M}^{\prime}$, shch that

$$
p_{n}(t)=t^{n}+\sum_{j=1}^{n} a_{j, M} t^{n-j}
$$

and

$$
q_{n}(t)=t^{n}+\sum_{j=1}^{n} a_{j, M}^{\prime} t^{n-j}
$$

satisfy

$$
\begin{gathered}
\left\|p_{n}\right\|_{M}=\min _{a_{j}}\left\|t^{n}+\sum_{j=1}^{n} a_{j} t^{n-j}\right\|_{M} ; \\
\left\|q_{n}\right\|_{(M)}=\min _{a_{j}}\left\|t^{n}+\sum_{j=1}^{n} a_{j} t^{n-j}\right\|_{(M)},
\end{gathered}
$$

here $\left\{a_{j, M}\right\}$ and $\left\{a_{j, M}^{\prime}\right\} \quad(j=1, \cdots, n)$ depend on $N$ function $M(u)$ corresponding to the Orlicz space.

2) The extremal functions $p_{n}(t)$ and $q_{n}(t)$ have $n$ different zero points in $(-1,1)$ respectively.

3) The odevity of extremal functions $p_{n}(t)$ and $q_{n}(t)$ is same to the odevity of natural number $n$.

Proof. 1) From [2] (pp. 160-168), we know, under the conditions of theorem 2, Orlicz spaces $L_{M}^{*}$ and $L_{(M)}^{*}$ are strictly convex. Since $H=\operatorname{span}\left\{1, t, \cdots, t^{n-1}\right\}$ is a finite dimensional linear subspace, (1) is obvious by the theory of best approximation (see [1], pp. 1-10).

2) From Theorem 1 we can easily obtain it.

3) Since $N$ function $M(u)$ is an even function, so

$$
\begin{aligned}
\left\|(-1)^{n} p_{n}(-t)\right\|_{M} & =\inf _{\alpha>0} \frac{1}{\alpha}\left(1+\int_{-1}^{1} M\left(\alpha(-1)^{n} p_{n}(-t)\right) \mathrm{d} t\right) \\
& =\inf _{\alpha>0} \frac{1}{\alpha}\left(1+\int_{-1}^{1} M\left(\alpha p_{n}(-t)\right) \mathrm{d} t\right) \\
& =\inf _{\alpha>0} \frac{1}{\alpha}\left(1+\int_{-1}^{1} M\left(\alpha p_{n}(t)\right) \mathrm{d} t\right) \\
& =\left\|p_{n}(t)\right\|_{M} .
\end{aligned}
$$

Analogously,

$$
\left\|(-1)^{n} q_{n}(-t)\right\|_{(M)}=\left\|q_{n}(t)\right\|_{(M)}
$$

holds true. Hence, from (1), the uniqueness of the extremal function, we obtain

$$
\begin{aligned}
& (-1)^{n} p_{n}(-t)=p_{n}(t) ; \\
& (-1)^{n} q_{n}(-t)=q_{n}(t) .
\end{aligned}
$$

By these, (3) follows.

\section{Acknowledgements}

This work is supported by the National Natural Science Foundation of China under the contracts No. 11161033 and the Natural Science Foundation of Inner Mongolia Autonomous Region under the contracts No. 2009MS0105.

\section{REFERENCES}

[1] Y. S. Sun, “Approximation Theory of Functions,” Beijing Normal University Press, Beijing, 1989.

[2] C. X. Wu and T. F. Wang, "Orlicz Space and Its Applications,” Hei Long Jiang Science and Technology Press, Harbin, 1983.

[3] D. L. Xie, "The Characteristics of Best Approximators in Orlicz Spaces,” Journal of Hangzhou University, Vol. 12, No. 3, 1985, pp. 319-322.

[4] Y. W. Wang and S. T. Chen, "The Best Approximating Operators in Orlicz Spaces,” Pure Mathematics and Applied Mathematics, Vol. 2, No. 2, 1986, pp. 44-51.

[5] C. X. Wu, T. F. Wang, S. T. Chen and Y. W. Wang, "The Geometric Theory of Orlicz Spaces,” Harbin Industrial University Press, Harbin, 1986.

[6] J. Gillis and G. Lewis, "Monic Polynomials with Minimal Norm,” Journal of Approximation Theory, Vol. 34, No. 2, 1982, pp. 187-193. doi:10.1016/0021-9045(82)90091-0 